\title{
Economic Burden of Switching to Different Biologic Therapies Among Tumor Necrosis Factor Inhibitor- Experienced Patients with Psoriatic Arthritis
}

\author{
Yan Song (D) - Keith A. Betts - Yichen Lu - Rakesh Singh • \\ Jerry Clewell · Jenny Griffith
}

Received: March 7, 2019 / Published online: May 4, 2019

(C) The Author(s) 2019

\begin{abstract}
Introduction: Patients with psoriatic arthritis (PsA) who receive an initial tumor necrosis factor inhibitor (TNFi) may switch to another TNFi or a non-TNFi biologic therapy. This study compared the healthcare resource use (HRU), expenditures, and time to discontinuation among TNFi-experienced patients with PsA who switched to different biologic therapies in the United States (US).

Methods: Adults with PsA who discontinued an initial TNFi (adalimumab, etanercept, infliximab, golimumab, or certolizumab pegol) and switched to another TNFi or a non-TNFi (ustekinumab or secukinumab) were identified in the Symphony Health Solutions database [Quarter (Q)1 2010-Q2 2017]. Eligible patients had claims data activity for $\geq 12$ months before (baseline) and after (study period) the switching date. All-cause HRU, costs (2017 US dollars), and time to discontinuation during the study period were compared between patients switching to another TNFi vs. a non-TNFi
\end{abstract}

Enhanced Digital Features To view enhanced digital features for this article go to: https://doi.org/10.6084/ m9.figshare.8026364.

Y. Song $(\triangle) \cdot$ K. A. Betts · Y. Lu

Analysis Group, Boston, MA, USA

e-mail: yan.song@analysisgroup.com

R. Singh $\cdot$ J. Clewell $\cdot$ J. Griffith

AbbVie Inc., North Chicago, IL, USA (index drug). Multivariable regression models adjusted for baseline covariates (index year, age, sex, initial TNFi, comorbidities, baseline HRU, and PsA-related treatment history).

Results: Of 2107 patients switching to another TNFi and 253 switching to a non-TNFi, adalimumab and etanercept were the most common initial TNFi in both cohorts. During the study period, patients switching to another TNFi had significantly fewer dermatologists visits $(0.43$; $p<0.01)$ but more rheumatologist visits $(1.56$, $p<0.01)$ than patients switching to a nonTNFi. Patients switching to another TNFi vs. a non-TNFi incurred significantly lower total average healthcare expenditures (adjusted difference: $\$ 17,625 ; p<0.01)$, driven by lower prescription drug (adjusted difference: $\$ 17,172$; $p<0.01$ ) and hospitalization expenditures (adjusted difference: $\$ 5772 ; p=0.04$ ). Patients who switched to another TNFi vs. a non-TNFi continued on their index therapy significantly longer (median time to discontinuation: 8.31 vs. 5.68 months; log-rank $p<0.01$ ).

Conclusions: Patients with PsA who switched to another TNFi had lower total healthcare expenditures and longer persistence compared with patients who switched to a non-TNFi biologic.

Funding: AbbVie.

Keywords: Claims database analysis;

Healthcare costs; Healthcare resource utilization; Psoriatic arthritis; Treatment 
discontinuation; Tumor necrosis factor inhibitor

\section{INTRODUCTION}

Psoriatic arthritis (PsA) is a chronic and progressive form of inflammatory arthritis associated with psoriasis $[1,2]$. The overall prevalence of PsA in the general population is approximately 133 per 100,000 people [3], although estimates have varied depending on location and disease definitions, and it affects men and women approximately equally $[4,5]$. Symptoms of PsA include inflammation, pain, and stiffness in the joints, and may result in significant, permanent joint damage, disability, reduced quality of life, or increased work-related issues $[2,6,7]$.

Multiple options are available for the treatment of active PsA, including nonsteroidal antiinflammatory drugs (NSAIDs), conventional synthetic disease-modifying anti-rheumatic drugs (DMARDs), and biologic therapies such as tumor necrosis factor- $\alpha$ inhibitors (TNFi; e.g., adalimumab, certolizumab pegol, etanercept, golimumab, and infliximab) [8, 9]. Recently, additional new therapies for PsA have been introduced to the market, including an oral phosphodiesterase 4 inhibitor (i.e., apremilast) [10] and targeted immunomodulators that interact with pathways other than TNF- $\alpha$ (e.g., abatacept, ixekizumab, secukinumab, ustekinumab, and tofacitinib) [11-14]. The overall goals of treatment are to control inflammation, reduce pain and discomfort, and prevent joint degeneration and disability [15].

In November 2017, the American College of Rheumatology (ACR) and National Psoriasis Foundation (NPF) issued draft guidelines for the treatment of active PsA that support the preferred use of TNFi among treatment-naïve patients [16]. Additionally, these guidelines favor the sequential use of a second TNFi over other biologic options in patients with an inadequate response to their initial TNFi. These draft recommendations are noted to be conditional based on a low threshold of evidence. Reddy et al. performed a systematic literature review on the real-world effectiveness of subsequent TNFi in refractory PsA and reported 18 eligible studies, with a general trend of decreasing effectiveness in later lines [9]. This finding is also confirmed in a 2017 literature review by Costa et al. [17]; however, the number of included studies was also low. To date, there have been no studies assessing the economic impact of switching from an initial TNFi to an alternative TNFi or non-TNFi biologic in PsA.

To address this gap in the literature, the present analysis aimed to compare the all-cause and PsA-related healthcare resource utilization (HRU) and expenditures of patients with PsA who discontinued their initial TNFi and switched to another TNFi or a non-TNFi biologic using a large United States (US)-based claims database. In addition, this study compared the time to discontinuation of second treatment among patients with PsA who discontinued the initial TNFi and switched to another TNFi vs. a non-TNFi biologic.

\section{METHODS}

\section{Data Source}

The data for this analysis were derived from the Symphony Health Solutions database (Q1 2010-Q2 2017), an insurance claims database that covers a range of payment types (e.g., cash, Medicaid, Medicare, and commercial insurance payments) and is representative of the demographics of the US in terms of age and sex distribution. This database links healthcare data for about 274 million people of the US population from three basic sources: pharmacy pointof-service sales, switch/network (clearing house) transactions, and additional direct prescriptions, medical, and hospital claims data feeds [18]. The database reflects pharmacy claims in all stages of processing, submitted medical claims, and includes physician National Provider Identifier numbers for the prescribing physician. Expenditure information for each pharmacy claim was estimated through linking the appropriate identification code in the claim to external source on medical charges (e.g., average wholesale price $[A W P][19,20])$. 
All data collected from the database are deidentified in compliance with the patient confidentiality requirement of the Health Insurance Portability and Accountability Act (HIPAA) and the Helsinki Declaration. Thus, no institutional board review was required.

\section{Study Population}

\section{Inclusion Criteria}

Sample selection criteria are described in Fig. 1. Patients with PsA who switched from an initial TNFi (adalimumab, etanercept, infliximab, golimumab, or certolizumab pegol) to a second TNFi or non-TNFi were included in the analysis. Eligible second TNFi therapies included adalimumab, etanercept, infliximab, golimumab, or certolizumab pegol, while non-TNFi biologics included ustekinumab or secukinumab. The date of the first prescription fill for this second agent (after January 1, 2012) was the index date. The biologic prescribed on this date was the index drug. Specifically, patients were included in the analysis if they: (1) were aged $\geq 18$ years as of the index date (defined below), (2) had continuous claims data activity (defined as $\geq 1$ pharmacy claim or $\geq 1$ medical claim every 3 months in the prescription drug database or medical database) during the 12-month period prior to the index date (baseline period) and during the 12-month period following the index date (study period), and (3) had $\geq 2$ diagnoses of PsA identified using International Statistical Classification of Diseases (ICD), 9th/ 10th edition (ICD-9 and ICD-10) codes 696.0 and L40.5, respectively, on distinct days. In addition, patients were required to have $\geq 2$ prescription fills for a TNFi on or after the first observed diagnosis of PsA with a continuous treatment course of at least 28 days. The first TNFi that met this criterion was defined as the initial TNFi. The first prescription of the initial TNFi was required to occur after the US Food and Drug Administration (FDA) approval date for treating PsA with the specific agent. Eligible patients also had $\geq 2$ prescription fills for a biologic different from the initial TNFi within 30 days before to 60 days after the discontinuation date of initial TNFi treatment, and after the FDA approval date for the specific agent. Eligible patients were divided into two cohorts: patients switching to a non-TNFi and those switching to another TNFi.

\section{Exclusion Criteria}

Patients were excluded from the analysis if they had: (1) any prescription for any targeted treatment (i.e., TNFi, non-TNFi, or apremilast) indicated for PsA before the first claim of the initial TNFi; (2) any use of the index drug before the index date; (3) more than one switched-to drug on the index date; or (4) any diagnoses of rheumatoid arthritis, ankylosing spondylitis, Crohn's disease, ulcerative colitis, or non-infectious uveitis before the index date.

\section{Study Outcomes}

Demographics (i.e., age, sex, year of the index date) were measured as of the index date, and Charlson Comorbidity Index (CCI) [21], PsArelated treatment history, HRU, and medical service expenditures were measured during the baseline period.

All-cause and PsA-related HRU (defined as claims for medical services associated with a diagnosis for PsA) were measured during the study period. The number of inpatient admissions (including total length of stay), outpatient visits (dermatologist, rheumatologist, and other outpatient visits), emergency room (ER) visits, and other visits (including lab tests, home health, etc.) were recorded. In addition, mean all-cause healthcare expenditures were measured during the study period (in 2017 USD) and included prescription drug expenditures (e.g., those associated with and not associated with the index drug) and medical services expenditure.

Time to discontinuation, defined as a gap of $\geq 60$ consecutive days with no medication supply (i.e., no fills of the index drug in the 60 days following the last day of supply of the index drug), was also measured. Patients were followed until treatment discontinuation or end of claims data activity (defined as the date of the last medical or drug claim in the database 


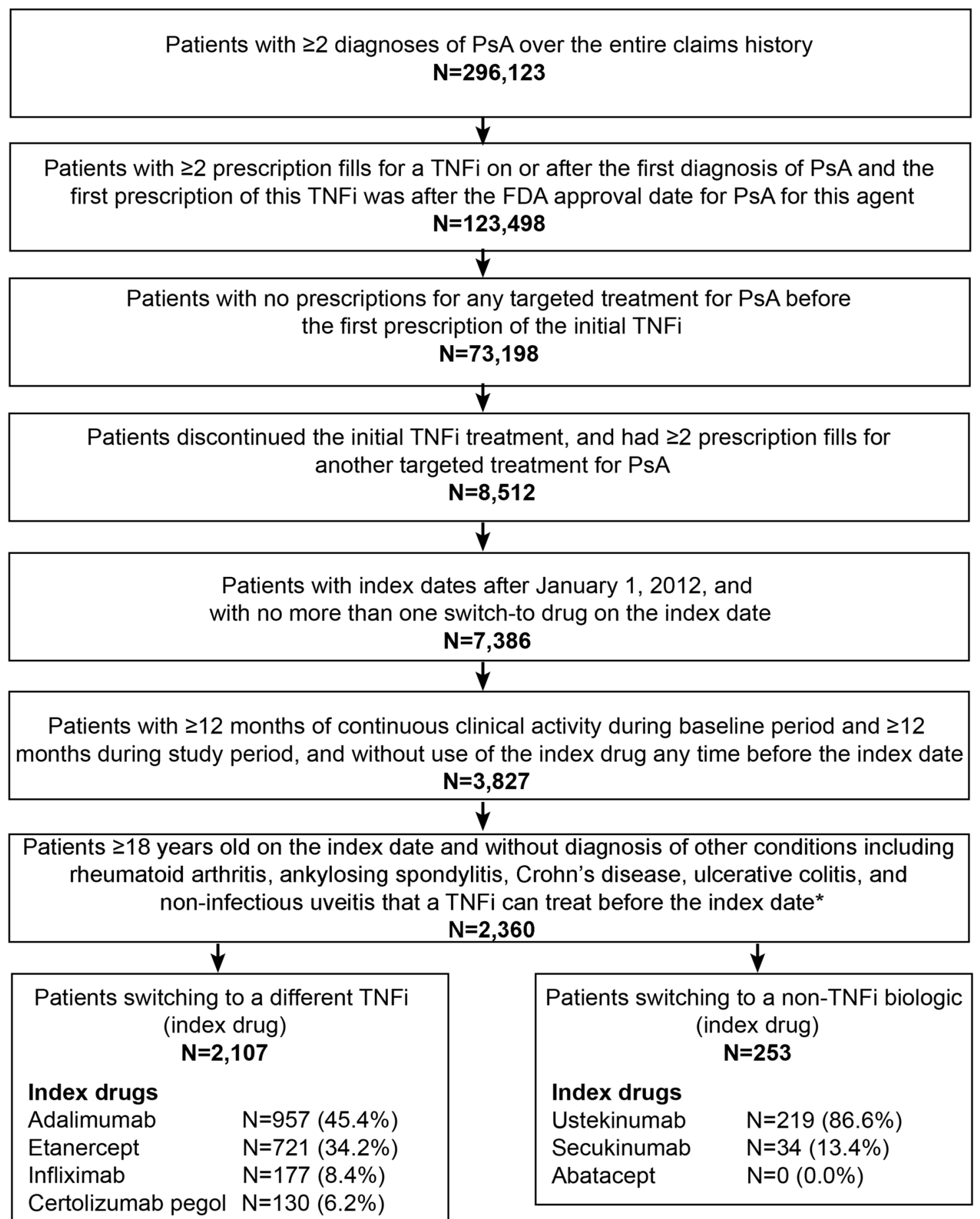

Fig. 1 Sample selection. FDA United States Food and Drug Administration, $P_{S} A$ psoriatic arthritis, TNFi tumor necrosis factor inhibitor. ${ }^{*}$ Patients $(N=231)$ who switched to apremilast after the initial TNFi were excluded from the analysis 
for a particular patient), whichever occurred first.

\section{Data Analysis}

Patient characteristics, comorbidities, all-cause HRU, and expenditures during the baseline period were summarized using descriptive statistics. Categorical baseline characteristics were compared using Chi-squared tests, and continuous baseline characteristics were compared using Wilcoxon rank-sum tests.

For HRU during the study period, unadjusted and adjusted incidence rate ratios (IRR) with 95\% confidence intervals (CI) for all-cause and PsA-related HRU rates, including inpatient admissions and outpatient, ER, and other visits, were estimated using negative binomial regression models. For binary HRU variables, odds ratios (OR) were estimated using logistic regression models.

Unadjusted all-cause and PsA-related healthcare expenditures, including medical and pharmacy expenditures, were compared using Wilcoxon rank-sum tests. Adjusted expenditures were estimated using a generalized linear model with Tweedie distribution.

Kaplan-Meier analysis was used to describe time to discontinuation between patients who switched to another TNFi and patients who switched to a non-TNFi biologic, and adjusted hazard ratios (HR) were estimated using Cox proportional hazards models. Covariates in the adjusted models included age, sex, year of index date, CCI, non-biologic DMARDs, systemic corticosteroids and pain medication received during baseline period, initial TNFi agent, number of all-cause inpatient admissions during baseline period, and number of all-cause ER visits during the baseline period.

All statistical analyses were conducted in SAS (version 9.4), and statistical significance was set at $p<0.05$.

\section{RESULTS}

Of 2591 eligible patients with PsA who discontinued their initial TNFi, 2107 switched to a different TNFi and 253 switched to a non-TNFi biologic (Fig. 1). The most frequently switchedto TNFi biologics were adalimumab $(45.4 \%)$ and etanercept (34.2\%), while ustekinumab (86.6\%) was the most frequently switched-to non-TNFi biologic. During the baseline period, the cohorts' distributions of age (mean, 50.09 [TNFi] vs. 51.35 years [non-TNFi]), sex (54.5 vs. $60.3 \%$ female, respectively), and CCI (mean [standard deviation (SD)]: 0.31 [0.79] vs. 0.39 [0.83]) were similar (Table 1).

Adalimumab (42.2 [TNFi] vs. 61.3\% [nonTNFi]; $p<0.05$ ) and etanercept (48.2 vs. $26.9 \%$, respectively; $p<0.05$ ) were the most common initial TNFis in both cohorts. Patients switching to another TNFi had higher use of non-biologic DMARDs (58.4\% vs. $46.2 \%$, respectively), systemic corticosteroids (57.7 vs. $48.6 \%$ ), and pain medications (71.9 vs. $65.6 \%$; all $p<0.05)$ at baseline compared with those switching to a non-TNFi. During the baseline period, HRU and expenditures were not significantly different between patients switching to another TNFi and those switching to a non-TNFi (Table 2). The percentages of patients with any inpatient admission were $6.9 \%$ for patients who switched to another TNFi and $8.3 \%$ for those who switched to a non-TNFi biologic; the percentages with outpatient visits were 10.0 and 9.2\%, respectively. The mean (SD) total all-cause medical services expenditures during the baseline period were $\$ 13,307(\$ 38,691)$ and $\$ 16,240$ $(\$ 35,374)$ for patients who switched to another TNFi or non-TNFi biologic, respectively. The difference between the two cohorts in baseline all-cause medical services expenditures was not statistically significant.

During the study period, patients who switched to another TNFi had numerically fewer allcause inpatient admissions (adjusted IRR [95\% CI] $0.85[0.51,1.40] ; p=0.52)$ and shorter hospitalization days $(0.63[0.30,1.30] ; p=0.21)$ compared with those who switched to nonTNFi, although the differences were not statistically significant (Table 3 ).

The numbers of all-cause ER (adjusted IRR [95\% CI] $0.97[0.66,1.41] ; p=0.87)$ and other visits $(0.94[0.71,1.24] ; p=0.64)$ were comparable between the two cohorts. However, patients switching to another TNFi had significantly fewer all-cause dermatologists visits 
Table 1 Patient baseline characteristics and treatment history

\begin{tabular}{|c|c|c|}
\hline Characteristics & $\begin{array}{l}\text { TNFi } \\
N=2107\end{array}$ & $\begin{array}{l}\text { Non-TNFi biologic } \\
N=253\end{array}$ \\
\hline Age, mean (SD) & $50.09(11.53)$ & $51.35(11.75)$ \\
\hline Female, $n(\%)$ & $1270(60.3 \%)$ & $138(54.5 \%)$ \\
\hline \multicolumn{3}{|l|}{ Year of index date, $n(\%)$} \\
\hline 2012 & $428(20.3 \%)$ & - \\
\hline 2013 & $510(24.2 \%)$ & $19(7.5 \%)^{*}$ \\
\hline 2014 & $456(21.6 \%)$ & $72(28.5 \%)^{*}$ \\
\hline 2015 & $496(23.5 \%)$ & $79(31.2 \%)^{*}$ \\
\hline 2016 & $217(10.3 \%)$ & $83(32.8 \%)^{*}$ \\
\hline $\mathrm{CCI}$, mean $(\mathrm{SD})$ & $0.31(0.79)$ & $0.39(0.83)$ \\
\hline \multicolumn{3}{|l|}{ Initial TNFi agent, $n(\%)$} \\
\hline Adalimumab & $890(42.2 \%)$ & $155(61.3 \%)^{*}$ \\
\hline Etanercept & $1015(48.2 \%)$ & $68(26.9 \%)^{*}$ \\
\hline Infliximab & $114(5.4 \%)$ & $16(6.3 \%)$ \\
\hline Golimumab & $64(3.0 \%)$ & $11(4.3 \%)$ \\
\hline Certolizumab pegol & $24(1.1 \%)$ & $3(1.2 \%)$ \\
\hline \multicolumn{3}{|c|}{ Other PsA-related treatments during baseline period, $n(\%)$} \\
\hline Non-biologic DMARDs & $1230(58.4 \%)$ & $117(46.2 \%)^{*}$ \\
\hline Systemic corticosteroids & $1216(57.7 \%)$ & $123(48.6 \%)^{*}$ \\
\hline Pain medications & $1515(71.9 \%)$ & $166(65.6 \%)^{*}$ \\
\hline
\end{tabular}

CCI Charlson Comorbidity Index, DMARD disease-modifying anti-rheumatic drug, $P s A$ psoriatic arthritis, $S D$ standard deviation, $T N F i$ tumor necrosis factor inhibitor

${ }^{*} p<0.05$

(adjusted IRR [95\% CI $\quad 0.43 \quad[0.28, \quad 0.66]$; $p<0.01$ ), but more all-cause rheumatologist visits $(1.56[1.22,2.00] ; p<0.01)$ than patients switching to a non-TNFi (Table 3). Similar results were observed in the analysis of PsA-related HRU among the two cohorts. Patients switching to another TNFi had significantly more PsA-related outpatient visits (adjusted IRR $[95 \% \mathrm{CI}] 1.34 \quad[1.11,1.60] ; p<0.01)$ and rheumatologist visits $(1.53 \quad[1.19,1.98]$; $p<0.01)$ than patients switching to a non-TNFi (Table 4). All other categories of PsA-related HRU during the study period were similar between the cohorts.
During the study period, patients switching to another TNFi incurred significantly lower total all-cause healthcare expenditures compared with patients who switched to a non-TNFi biologic (mean expenditures: $\$ 69,588$ [TNFi] vs. $\$ 84,225$ [non-TNFi]; adjusted difference: $\$ 17,625 ; p<0.01$ ) (Fig. 2). This difference was primarily driven by lower prescription drug expenditures (adjusted difference: \$17,172; $p<0.01$ ), particularly with regards to the expenditures for the index drug (mean expenditures: $\$ 40,468$ [TNFi] vs. $\$ 57,963$ [non-TNFi]). The total all-cause medical service expenditures were comparable between the cohorts (adjusted 
Table 2 HRU and healthcare costs (2017 USD) during the 12-month baseline period

\begin{tabular}{lrr}
\hline & TNFi & $\begin{array}{c}\text { Non-TNFi biologic } \\
N=253\end{array}$ \\
\hline Total all-cause HRU during the baseline period & $\mathbf{N = 2 1 0 7}$ & $21(8.3 \%)$ \\
Any inpatient admission, $n(\%)$ & $146(6.9 \%)$ & $0.10(0.35)$ \\
Number of inpatient admissions, mean (SD) & $0.09(0.38)$ & $9.74(9.18)$ \\
Number of outpatient visits, mean (SD) & $10.10(10.00)$ & $0.26(0.85)$ \\
Number of emergency room visits, mean (SD) & $0.25(0.83)$ & $1.70(3.36)$ \\
Other visits, mean (SD) & $1.36(2.71)$ & $16,240(35,374)$ \\
Total all-cause medical service costs during the baseline period, mean (SD) & $13,307(38,691)$ \\
Inpatient stays costs & $1880(17,690)$ & $3123(18,763)$ \\
Outpatient visits costs & $10,322(32,199)$ & $10,861(26,852)$ \\
Emergency room visits costs & $311(1194)$ & $303(1057)$ \\
Other visits costs & $793(3580)$ & $1953(11,971)$ \\
\hline
\end{tabular}

$H R U$ health resource utilization, SD standard deviation, TNFi tumor necrosis factor inhibitor, USD United States dollars

Table 3 All-cause HRU during the 12-month study period stratified by TNFi and non-TNFi biologic users

\begin{tabular}{|c|c|c|c|c|c|c|}
\hline Healthcare resource & $\begin{array}{l}\text { TNFi } \\
N=2107\end{array}$ & $\begin{array}{l}\text { Non-TNFi biologic } \\
N=253\end{array}$ & Unadjusted com & arison & Adjusted compa & son \\
\hline Numbers of events per & patient per & ear & IRR (95\% CI) & $P$ & IRR (95\% CI) & $\boldsymbol{P}$ \\
\hline Inpatient admissions & 0.14 & 0.14 & $1.02(0.62,1.67)$ & 0.94 & $0.85(0.51,1.40)$ & 0.52 \\
\hline Inpatient days & 0.53 & 0.58 & $0.92(0.45,1.87)$ & 0.81 & $0.63(0.30,1.30)$ & 0.21 \\
\hline Outpatient visits & 9.83 & 8.67 & $1.13(0.99,1.30)$ & 0.07 & $1.08(0.95,1.24)$ & 0.25 \\
\hline Dermatologist & 0.52 & 1.04 & $0.50(0.33,0.76)$ & $<0.01^{*}$ & $0.43(0.28,0.66)$ & $<0.01^{*}$ \\
\hline Rheumatologist & 2.24 & 1.30 & $1.72(1.35,2.19)$ & $<0.01^{*}$ & $1.56(1.22,2.00)$ & $<0.01^{*}$ \\
\hline Other visits & 7.07 & 6.33 & $1.12(0.95,1.31)$ & 0.18 & $1.09(0.93,1.28)$ & 0.30 \\
\hline Emergency room visits & 0.26 & 0.25 & $1.04(0.70,1.54)$ & 0.84 & $0.97(0.66,1.41)$ & 0.87 \\
\hline Other visits & 1.33 & 1.62 & $0.82(0.63,1.08)$ & 0.16 & $0.94(0.71,1.24)$ & 0.64 \\
\hline Any use (\% of patients & & OR & $95 \% \mathrm{CI})$ & $\boldsymbol{P}$ & OR $(95 \% \mathrm{CI})$ & $\boldsymbol{P}$ \\
\hline Any inpatient admission & $9.3 \%$ & $9.9 \%$ & $0.60,1.45)$ & 0.77 & $0.91(0.57,1.47)$ & 0.70 \\
\hline
\end{tabular}

$C I$ confidence interval, $H R U$ health resource utilization; IRR incidence rate ratio, $O R$ odds ratio, TNFi tumor necrosis factor inhibitor

${ }^{*} p<0.05$

difference: $\$ 141 ; p=0.97)$, although patients switching to another TNFi had significantly lower expenditures for all-cause inpatient admissions compared with those switching to a
non-TNFi (adjusted difference: $\$ 5772 ; p=0.04$ ). All-cause healthcare expenditures for ER, outpatient, and other services were comparable between the cohorts. 
Table 4 PsA-related HRU during the 12-month study period stratified by TNFi and non-TNFi biologic users

\begin{tabular}{|c|c|c|c|c|c|c|}
\hline & TNFi & $\begin{array}{l}\text { Non-TNFi biologic } \\
N=253\end{array}$ & Unadjusted com & arison & Adjusted compa & son \\
\hline Numbers of events per & atient per $y$ & & IRR (95\% CI) & $P$ & IRR (95\% CI) & $P$ \\
\hline Inpatient admissions & 0.04 & 0.04 & $1.08(0.48,2.42)$ & 0.85 & $0.76(0.33,1.76)$ & 0.53 \\
\hline Inpatient days & 0.20 & 0.15 & $1.32(0.37,4.75)$ & 0.67 & $0.96(0.24,3.87)$ & 0.96 \\
\hline Outpatient visits & 3.21 & 2.14 & $1.50(1.25,1.79)$ & $<0.01^{*}$ & $1.34(1.11,1.60)$ & $<0.01^{*}$ \\
\hline Dermatologist & 0.11 & 0.17 & $0.62(0.28,1.42)$ & 0.26 & $0.75(0.32,1.75)$ & 0.50 \\
\hline Rheumatologist & 1.94 & 1.18 & $1.65(1.29,2.10)$ & $<0.01^{*}$ & $1.53(1.19,1.98)$ & $<0.01^{*}$ \\
\hline Other outpatient visits & 1.16 & 0.79 & $1.46(1.10,1.95)$ & $0.01^{*}$ & $1.26(0.94,1.69)$ & 0.13 \\
\hline Emergency room visits & 0.03 & 0.02 & $1.46(0.56,3.84)$ & 0.44 & $1.14(0.42,3.06)$ & 0.80 \\
\hline Other visits & 0.31 & 0.23 & $1.37(0.84,2.23)$ & 0.21 & $1.41(0.85,2.34)$ & 0.19 \\
\hline Any use (\% of patients) & & OR & $\% \mathrm{CI})$ & & OR $(95 \% \mathrm{CI})$ & $P$ \\
\hline Any inpatient admission & $3.2 \%$ & $3.2 \%$ & $0.48,2.12)$ & .99 & $0.87(0.39,1.92)$ & 0.72 \\
\hline
\end{tabular}

$C I$ confidence interval, $H R U$ health resource utilization, $I R R$ incidence rate, $O R$ odds ratio, $P s A$ psoriatic arthritis, $T N F i$ tumor necrosis factor inhibitor ${ }^{*} p<0.05$

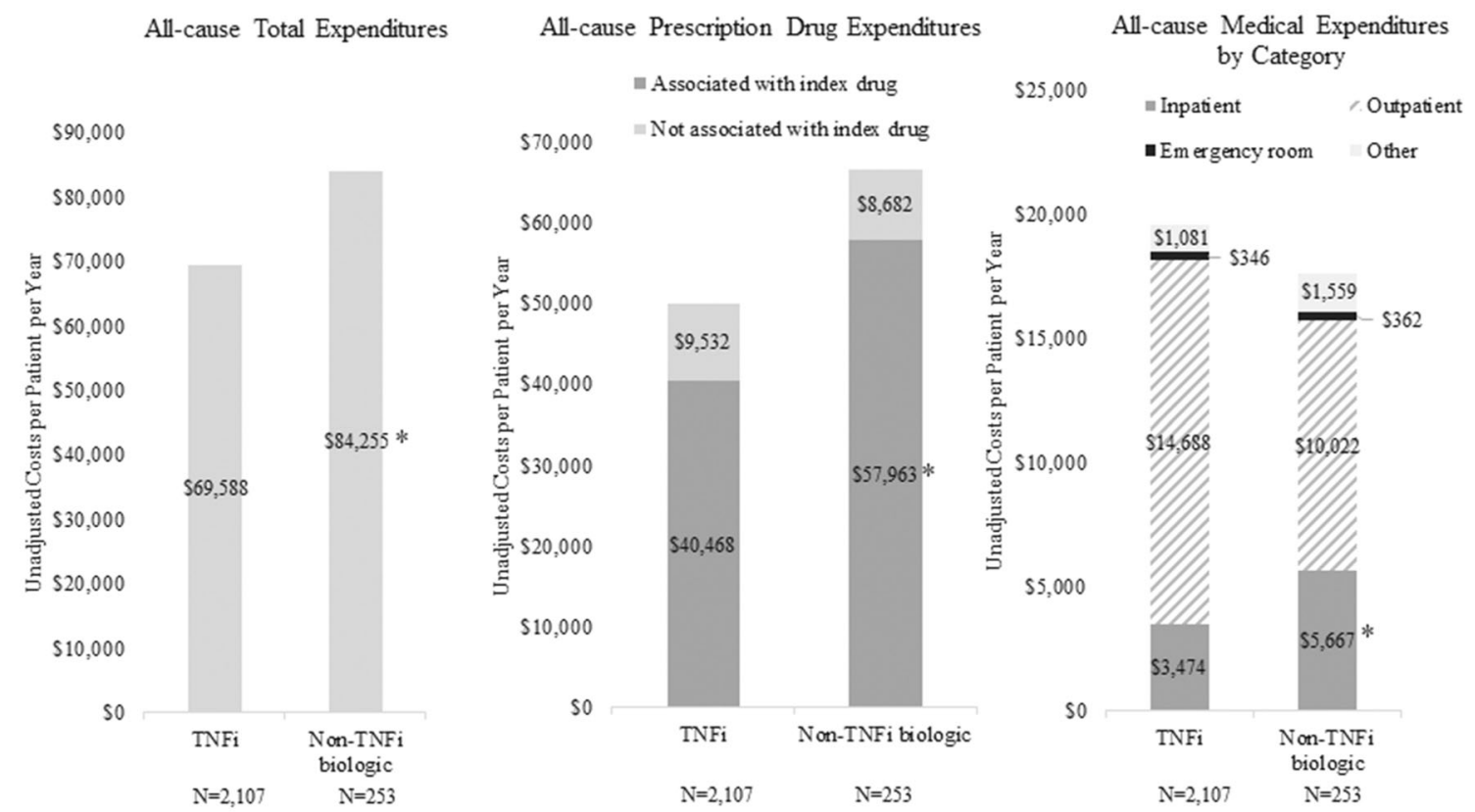

Fig. 2 All-cause healthcare expenditures (2017 USD) during the 12-month study period. TNFi tumor necrosis factor inhibitor, USD United States dollars. ${ }^{*} p<0.05$

With regards to PsA-related healthcare expenditures during the study period, patients switching to another TNFi had significantly lower total PsA-related healthcare expenditures (mean expenditures: $\$ 59,261$ [TNFi] vs. $\$ 70,882$ [non-TNFi]; adjusted difference: \$13,375; 
$p<0.01$ ) (Fig. 3) and prescription drug expenditures (adjusted difference: $\$ 16,660 ; p<0.01$ ) compared with those switching to a non-TNFi biologic. However, patients switching to another TNFi had significantly higher outpatient expenditures compared with patients switching to non-TNFi biologics (adjusted difference: $\$ 3565 ; p=0.02$ ).

In the Kaplan-Meier analysis of time to discontinuation, patients who switched to another TNFi continued on therapy significantly longer compared with those who switched to a nonTNF biologic (median time to discontinuation: 8.31 vs. 5.68 months, respectively; log-rank $p<0.01$ ) (Fig. 4). The results of the adjusted analysis showed a similar trend, with a significantly higher rate of discontinuation in the non-TNF cohort compared with the TNFi cohort (HR [95\% CI] $1.35[1.16,1.56] ; p<0.01)$.

\section{DISCUSSION}

This real-world US administrative claims database analysis compared the HRU, expenditures, and time to discontinuation among TNFi-experienced patients with PsA who switched to different subsequent biologic therapies for any reason. The results indicated that patients with PsA who switched to another TNFi after discontinuation of the initial TNFi incurred lower total healthcare expenditures, mainly driven by lower prescription drug expenditures, compared with patients who switched to a non-TNFi biologic. During the study period, patients switching to another TNFi also had significantly more all-cause and PsA-related outpatient visits to rheumatologists compared with those switching to a non-TNFi biologic. Conversely, patients switching to a non-TNFi biologic had significantly more all-cause and numerically more PsA-related visits to a dermatologist than those who switched to another TNFi. The difference in the number of physician visits between the two patient groups could potentially reflect a preference for using a second TNFi among rheumatologists vs. dermatologists, although this is to be further investigated. The total medical service expenditures were comparable between cohorts, although the TNFi cohort had significantly lower all-cause hospitalization expenditures than the non-TNFi cohort. These findings suggest potential economic benefits for TNFi-experienced patients
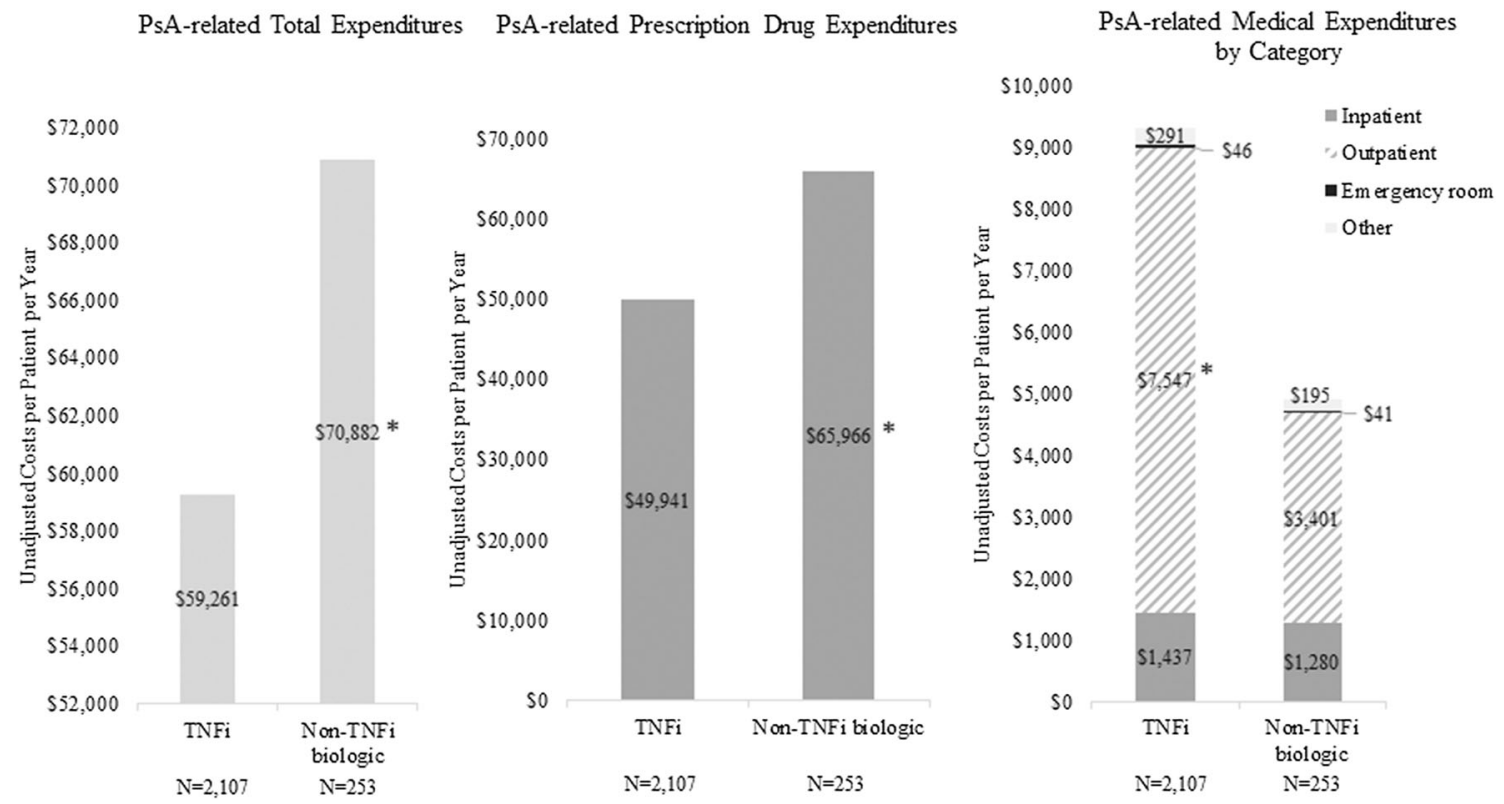

Fig. 3 PsA-related healthcare expenditures (2017 USD) during the 12-month study period. PsA psoriatic arthritis, TNFi tumor necrosis factor inhibitor, USD United States dollars. ${ }^{*} p<0.05$ 


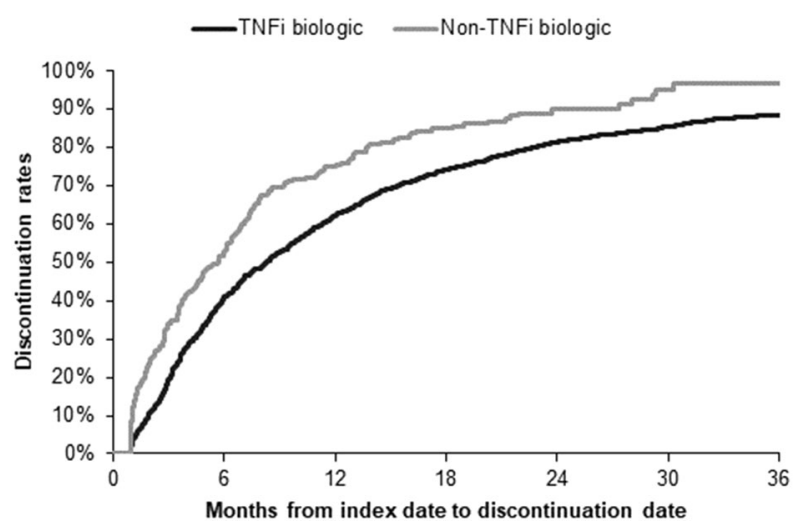

Fig. 4 Kaplan-Meier analysis of time to discontinuation of the index drug. TNFi tumor necrosis factor inhibitor. ${ }^{*} \log$-rank $p<0.05$

with refractory PsA who initiate a subsequent TNFi vs. a non-TNFi biologic.

In addition, the current study showed that patients who switched to another TNFi had longer treatment duration than patients who switched to a non-TNFi biologic. Previous research has reported good long-term persistence among patients with PsA receiving a TNFi for both the first and subsequent treatment [22]. Patients may discontinue TNFi or non-TNFi biologics due to various medical and non-medical reasons, including lack of efficacy and intolerable adverse events [23]. However, it is challenging to identify the reason of discontinuation for each patient in the current study due to the limitations of administrative claims data. Future studies using data sources with more clinical information (e.g., through a medical chart review) are warranted to better describe the reasons for the higher discontinuation rates associated with non-TNFi biologics compared with those associated with TNFis for the treatment of PsA.

To date, traditional PsA treatments (e.g., NSAIDs and conventional synthetic DMARDs) have not demonstrated the ability to slow the radiographic progression of joint disease, but anti-TNF agents have shown to be effective in this regard [11]. Radiographically evidenced protection of joint structure has become a goal of treatment and anti-TNFi agents are listed as the preferred PsA treatments among treatmentnaïve patients in the draft guidelines by the ACR and NPF [16]. Many therapeutic agents are currently available for PsA, and the current guidelines for the order of drug use in cases of inefficacy or toxicity of the first-line TNFi are not consistent. Recent clinical guidelines for PsA include recommendations published in 2016 by the European League Against Rheumatism (EULAR) and the Group for Research and Assessment of Psoriasis and Psoriatic Arthritis (GRAPPA) [24]. EULAR proposed a sequential treatment scheme in the case of adverse prognostic factors, suggesting that the initial TNFi be followed by non-TNFi agents after each respective treatment option is contraindicated. In contrast, the GRAPPA guidelines recommend the two treatment options (second TNFi and non-TNFi) alongside, allowing the physicians to choose the therapy depending on prognostic factors, comorbidities, availability of therapy, and patient preference [24]. In the latest draft guidelines by the ACR and NPF, sequential use of a second TNFi was recommended over other biologic options in patients with an inadequate response to the initial TNFi. However, these draft recommendations are noted to be conditional based on a low threshold of evidence [16], and thus further real-world studies are needed.

Several observational studies have been conducted to evaluate response for the initial and second-line TNFi for patients with PsA $[25,26]$. In an observational study using data from five Norwegian centers, patients who started their initial TNFi and switched to a second TNFi had a lower response rate compared 
with the response rate to the initial TNFi, both among themselves as well as the non-switchers [25]. On the other hand, a survey study in England reported that the majority of patients showed adequate response to a second- or thirdline biologic, and recommended guidelines to allow more therapeutic alternatives for the refractory cases [26]. However, to date, little real-world evidence has been published comparing the outcomes, including HRU and healthcare expenditures, between patients with PsA who switched to another TNFi vs. non-TNFi after the failure of the initial TNFi treatment. Thus, the current study provides the first realworld evidence of these economic impacts in PsA, contributing valuable insight to inform clinician and payer decisions in treatment selection.

\section{Limitations}

This study is subject to several limitations. First, no patients who switched to abatacept were identified in this analysis, potentially due to its recent introduction to the market. Second, the analysis did not include newly approved nonTNFi biologic agents (e.g., ixekizumab or tofacitinib), which were approved by FDA after the data cutoff. Thus, future studies should be conducted to evaluate the economic impact of these agents in PsA. Third, the Symphony database contained no eligibility records. Thus, continuous claims data activity was used as a surrogate for continuous eligibility, and the patient sample included might be different from the patient population in the real-world practice. Fourth, the medical claims recorded in the database may be incomplete as the Symphony database is not a closed system. Therefore, the actual costs incurred during the study period may be underestimated. Fifth, the pharmacy expenditure data were linked to an external source (AWP), which could be viewed as a standardized charge reference, but does not reflect actual reimbursed amounts.

Furthermore, this study is also subject to limitations common to claims analyses. For example, the presence of a claim for prescription does not indicate that the medication was consumed or that it was taken as prescribed. In addition, the results may be confounded from unavailable covariates such as clinical disease activity and clinical phenotype of patients. In this study, we adjusted baseline disease activity by controlling for baseline non-biologic DMARD use, systemic corticosteroid use, pain medication use, and baseline HRU in regression models. Admittedly, there may be residual confounding due to unobserved risk factors. However, given that the TNFi arm showed higher disease activity at baseline (as indicated by more use of non-biologic DMARDs, systemic corticosteroids, and pain medications), but showed lower healthcare expenditure in the study period, this study provides a conservative estimate of the difference in economic burden between the two cohorts. Finally, the results of this study may not be generalizable to patients outside of those represented in the Symphony Health Solutions database.

\section{CONCLUSIONS}

Adult patients with PsA who switched to another TNFi after discontinuation of their initial TNFi incurred lower total all-cause and PsA-related healthcare expenditures than patients who switched to a non-TNFi biologic. The cost difference was driven primarily by savings in both prescription drug and hospitalization expenditures among those patients switching to a second TNFi; on the other hand, the outpatient cost was higher among patients who switched to another TNFi. Additionally, patients who switched to another TNFi persisted longer on the therapy than patients who switched to a non-TNF biologic. These findings suggest potential overall economic savings associated with of switching to a different TNFi compared with switching to a non-TNFi biologic among TNFi-experienced patients with PsA.

\section{ACKNOWLEDGEMENTS}

Funding. Sponsorship for this study and article processing charges were funded by 
AbbVie. The sponsor was involved in all aspects of the study, drafting of the manuscript, and in the decision to submit the article for publication. All authors had full access to all of the data in this study and take complete responsibility for the integrity of the data and accuracy of the data analysis.

Medical Writing and Other Assistance. The authors would like to thank Shelley Batts, PhD from Analysis Group, Inc. for medical writing assistance and Wenxi Tang from Analysis Group, Inc. for analytical support. Financial support for these services was provided by AbbVie.

Authorship. All named authors meet the International Committee of Medical Journal Editors (ICMJE) criteria for authorship for this article, take responsibility for the integrity of the work as a whole, and have given their approval for this version to be published.

Disclosures. Yan Song is an employee of Analysis Group, Inc., which has received consulting fees from AbbVie. Keith A. Betts is an employee of Analysis Group, Inc., which has received consulting fees from AbbVie. Yichen $\mathrm{Lu}$ is an employee of Analysis Group, Inc., which has received consulting fees from AbbVie. Rakesh Singh is an employee of AbbVie and owns company stock. Jerry Clewell is an employee of AbbVie and owns company stock. Jenny Griffith is an employee of AbbVie and owns company stock.

Compliance with Ethics Guidelines. All data collected from the database are de-identified in compliance with the patient confidentiality requirement of the Health Insurance Portability and Accountability Act (HIPAA) and the Helsinki Declaration. Thus, no institutional board review was required.

Data Availability. The data that support the findings of this study are available from Symphony Health Solutions, Inc., but restrictions apply to the availability of these data, which were used under license for the current study, and so are not publicly available.
Open Access. This article is distributed under the terms of the Creative Commons Attribution-NonCommercial 4.0 International License (http://creativecommons.org/licenses/ by-nc/4.0/), which permits any noncommercial use, distribution, and reproduction in any medium, provided you give appropriate credit to the original author(s) and the source, provide a link to the Creative Commons license, and indicate if changes were made.

\section{REFERENCES}

1. Brockbank J, Gladman D. Diagnosis and management of psoriatic arthritis. Drugs. 2002;62(17):2447-57.

2. Christophers E, Barker JN, Griffiths CE, Dauden E, Milligan G, Molta $C$, et al. The risk of psoriatic arthritis remains constant following initial diagnosis of psoriasis among patients seen in European dermatology clinics. J Eur Acad Dermatol Venereol. 2010;24(5):548-54.

3. Scotti L, Franchi M, Marchesoni A, Corrao G. Prevalence and incidence of psoriatic arthritis: a systematic review and meta-analysis. Semin Arthritis Rheum. 2018;48(1):28-34.

4. Shbeeb M, Uramoto KM, Gibson LE, O'Fallon WM, Gabriel SE. The epidemiology of psoriatic arthritis in Olmsted County, Minnesota, USA, 1982-1991. J Rheumatol. 2000;27(5):1247-50.

5. Madland TM, Apalset EM, Johannessen AE, Rossebo B, Brun JG. Prevalence, disease manifestations, and treatment of psoriatic arthritis in western Norway. J Rheumatol. 2005;32(10):1918-22.

6. Gladman DD, Antoni C, Mease P, Clegg DO, Nash P. Psoriatic arthritis: epidemiology, clinical features, course, and outcome. Ann Rheum Dis. 2005;64(Suppl 2):ii14-7.

7. Taylor WJ. Impact of psoriatic arthritis on the patient: through the lens of the WHO International Classification of Functioning, Health, and Disability. Curr Rheumatol Rep. 2012;14(4):369-74.

8. Camussi G, Lupia E. The future role of anti-tumour necrosis factor (TNF) products in the treatment of rheumatoid arthritis. Drugs. 1998;55(5):613-20.

9. Reddy SM, Crean S, Martin AL, Burns MD, Palmer JB. Real-world effectiveness of anti-TNF switching 
in psoriatic arthritis: a systematic review of the literature. Clin Rheumatol. 2016;35(12):2955-66.

10. Torres T, Puig L. Apremilast: a novel oral treatment for psoriasis and psoriatic arthritis. Am J Clin Dermatol. 2018;19(1):23-32.

11. Raychaudhuri SP, Wilken R, Sukhov AC, Raychaudhuri SK, Maverakis E. Management of psoriatic arthritis: early diagnosis, monitoring of disease severity and cutting edge therapies. J Autoimmun. 2017;76:21-37.

12. Gladman D, Rigby W, Azevedo VF, Behrens F, Blanco R, Kaszuba A, et al. Tofacitinib for psoriatic arthritis in patients with an inadequate response to TNF inhibitors. N Engl J Med. 2017;377(16): 1525-36.

13. Toussirot E. Ixekizumab: an anti- IL-17A monoclonal antibody for the treatment of psoriatic arthritis. Expert Opin Biol Ther. 2018;18(1):101-7.

14. Roberts J, O'Rielly DD, Rahman P. A review of ustekinumab in the treatment of psoriatic arthritis. Immunotherapy. 2018;10(5):361-72.

15. Maharaj AB, Chandran V. Treatment of psoriatic arthritis with traditional DMARD's and novel therapies: approaches and recommendations. Expert Rev Clin Immunol. 2017;13(4):319-31.

16. Davio K. ACR and NPF unveil new clinical guideline for treating psoriatic arthritis. http://www.ajmc. com/conferences/acr-2017/acr-and-npf-unveil-newclinical-guideline-for-treating-psoriatic-arthritis. Accessed 7 May 2018.

17. Costa L, Perricone C, Chimenti MS, Del Puente A, Caso P, Peluso R, et al. Switching between biological treatments in psoriatic arthritis: a review of the evidence. Drugs R D. 2017;17(4):509-22.

18. Symphony Health Solutions. IDV (Integrated Dataverse) Product Introduction. https:// symphonyhealth.com/wp-content/uploads/2015/ 10/IDV-Product-Introduction.pdf. Accessed 16 May 2018.
19. Lee J, Pelkey R, Gubitosa J, Henrick MF, Ganz ML. Comparing healthcare costs associated with oral and subcutaneous methotrexate or biologic therapy for rheumatoid arthritis in the United States. Am Health Drug Benefits. 2017;10(1):42-9.

20. IBM Watson Health. RED BOOK- Average Wholesale Price. http://truvenhealth.com/products/ micromedex/product-suites/clinical-knowledge/redbook. Accessed 16 May 2018.

21. Quan H, Sundararajan V, Halfon P, Fong A, Burnand $\mathrm{B}$, Luthi JC, et al. Coding algorithms for defining comorbidities in ICD-9-CM and ICD-10 administrative data. Med Care. 2005;43(11): 1130-9.

22. Fagerli KM, Kearsley-Fleet L, Watson KD, Packham J, Symmons DPM, Contributors Group B-R, et al. Long-term persistence of TNF-inhibitor treatment in patients with psoriatic arthritis. Data from the British Society for Rheumatology Biologics Register. RMD Open. 2018;4(1):e000596.

23. Costa L, Perricone C, Chimenti MS, Del Puente A, Caso P, Peluso R, et al. Switching between biological treatments in psoriatic arthritis: a review of the evidence. Drugs R\&D. 2017;17(4):509-22.

24. Gossec L, Coates LC, de Wit M, Kavanaugh A, Ramiro S, Mease PJ, et al. Management of psoriatic arthritis in 2016: a comparison of EULAR and GRAPPA recommendations. Nat Rev Rheumatol. 2016;12(12):743-50.

25. Fagerli KM, Lie E, van der Heijde D, Heiberg MS, Kalstad S, Rodevand E, et al. Switching between TNF inhibitors in psoriatic arthritis: data from the NOR-DMARD study. Ann Rheum Dis. 2013;72(11): 1840-4.

26. Jani M, Macphie E, Rao C, Moore S, Mirjafari $H$, McLoughlin Y, et al. Effectiveness of switching between biologics in psoriatic arthritis- results of a large regional survey. Clin Med (Lond). 2014;14(1):95-6. 